\title{
Reflets
}

Revue ontaroise d'intervention sociale et communautaire

\section{Rapport du DÉFI '94: courage et volonté}

\section{Brigitte Nolet}

Volume 1, numéro 1, printemps 1995

Des pratiques à notre image : défis et ressources

URI : https://id.erudit.org/iderudit/026069ar

DOI : https://doi.org/10.7202/026069ar

Aller au sommaire du numéro

Éditeur(s)

Reflets : Revue ontaroise d'intervention sociale et communautaire

ISSN

1203-4576 (imprimé)

1712-8498 (numérique)

Découvrir la revue

Citer cet article

Nolet, B. (1995). Rapport du DÉFI '94: courage et volonté. Reflets, 1(1), 227-232.

https://doi.org/10.7202/026069ar

Tous droits réservés (C) Reflets : Revue ontaroise d'intervention sociale et communautaire, 1995

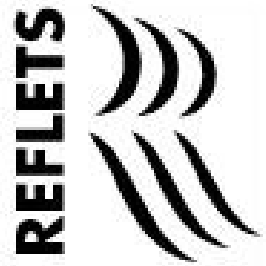

Ce document est protégé par la loi sur le droit d'auteur. L'utilisation des services d’Érudit (y compris la reproduction) est assujettie à sa politique d'utilisation que vous pouvez consulter en ligne.

https://apropos.erudit.org/fr/usagers/politique-dutilisation/ 


\section{Rapport du DÉFI'94}

\section{Courage et volonté}

\section{Brigitte Nolet}

Centre de santé communautaire du Niagara

J'ai l'impression d'avoir été une jeune fille ignorante des vrais problèmes sociaux avant de débuter un stage de travail au Centre de santé communautaire du Niagara en mai 1994. Cette expérience m'a permis de prendre conscience de l'omniprésence de certains problèmes sociaux, plus particulièrement au niveau de la violence, et de leur impact sur la vie quotidienne des gens. Ce stage a si bien piqué mon intérêt que j’ai décidé de diriger mes études universitaires vers le service social.

J'ai oeuvré au Centre de santé en tant que recherchiste. Mon travail consistait à recueillir de l'information sur les groupes de traitement pour hommes qui violentent leur conjointe, et de produire une bibliographie annotée, en y incluant les ressources répertoriées.

Cet article ne constitue pas une analyse des différents programmes existants, mais vise plutôt à explorer les caractéristiques contribuant à l'efficacité d'un groupe pour hommes violents. Mon analyse de ces caractéristiques s'appuie sur les opinions et les conseils que les intervenants et intervenantes ont bien voulu partager, ainsi que sur des réflexions et des observations découlant de ces quatre mois de recherche. 


\section{Philosophie}

Afin d'optimiser l'efficacité d'un groupe pour hommes violents, il faut d'abord que la philosophie sur laquelle se construit le groupe soit acceptée par tous les intervenants et intervenantes engagés dans sa mise en oeuvre. Cette philosophie doit être imprégnée d'une attitude positive et libre de tout stéréotype chauviniste. Le groupe doit développer des mécanismes pour remettre aux hommes la responsabilité de leurs actes; personne ne force un homme à être violent, il s'agit d'un choix qu'il fait et qu'il doit assumer. La colère ne peut pas servir de prétexte à des actes violents. De plus, lors des sessions de groupe, les hommes ont à remettre en question leurs croyances chauvinistes qui se manifestent, entre autres, par des besoins de contrôle, ainsi que certains stéréotypes face à leur perception du rôle masculin. Par contre, les intervenants et intervenantes doivent garder en tête que la socialisation constitue un déterminant majeur de la pensée de ces hommes, et que les efforts de changement au niveau des croyances sont difficiles et frustrants. L'homme violent croit qu'il a le devoir, le droit et le pouvoir de contrôler sa famille. Les techniques de contrôle de la colère (anger management) doivent faire partie des apprentissages de l'homme violent. Par contre, ces hommes ne font malheureusement souvent que transformer leur violence en d'autres moyens de contrôle, comme la manipulation par exemple. Cet aspect ne peut être négligé lors des sessions et le traitement doit composer avec sa volonté plus ou moins explicite de garder le contrôle sur sa famille. Il semble en effet difficile pour ces hommes de comprendre que la violence soit un signe de perte de ce contrôle qu'ils cherchent désespérément à garder. Si ces hommes acceptent que par leur participation, ils peuvent contribuer au développement du programme, peut-être seront-ils plus honnêtes envers eux-mêmes et envers le groupe, et plus en mesure de valoriser leur traitement et d'en tirer profit. 


\section{Le groupe}

Les groupes pour hommes violents s'avèrent efficaces lorsqu'ils sont axés sur une matière concrète qui réussit à rejoindre les hommes et à les aider. Je présenterai d'abord quelques programmes pour ensuite en décrire différents aspects qui contribuent, selon moi, au succès d'un groupe pour hommes violents. Lors de ma recherche, les programmes mentionnés les plus souvent sont le Domestic Abuse Intervention Project (DIAP) de Duluth au Minnesota et le manuel Learning to Live Without Violence écrit par Daniel Sonkin et al. Le programme Changing Ways établi à London (Ontario), ainsi que la série Men's Work produite par Paul Kivel, sont aussi renommés dans ce domaine. Une mise en commun des principaux éléments de ces quatre programmes fournit un ensemble de ressources très intéressantes.

D'abord, je considère qu'un programme efficace doit se baser sur une approche pro-féministe qui se concentre sur la sécurité des femmes violentées et sur l'emprise (empowerment). Le programme serait axé sur deux composantes, l'une éducative et l'autre thérapeutique. Cette dernière composante sera plus enrichissante si les hommes ont déjà participé à des sessions éducatives. Cette façon de fonctionner aurait aussi l'avantage de permettre la participation simultanée d'un plus grand nombre d'hommes, contribuant ainsi à diminuer les listes d'attente pour les programmes.

Dans ce modèle, la phase éducative pourrait comprendre 10 à 12 rencontres hebdomadaires qui seraient suivies d'une phase thérapeutique de 12 à 14 sessions s'échelonnant sur autant de semaines, selon les besoins du groupe. Une première rencontre d'évaluation avec l'homme (1 heure) avant qu'il soit accepté dans le programme ainsi qu'une session d'orientation (2 heures) qui explique le programme, ses buts et ses attentes compléteraient la structure de ce modèle.Vingt-cinq personnes pourraient participer à la phase éducative; une participation de 8 à 12 hommes serait optimale pour la phase thérapeutique. 
Reflets

Il est aussi important de mentionner quelques points susceptibles d'augmenter l'efficacité du programme. L'évaluation du groupe ainsi qu'un suivi des participants suite au groupe permettraient aux organismes de mieux connaître les points forts et faibles du programme afin de raffiner leur approche. Un bon programme devrait aussi toujours permettre aux intervenantes et intervenants de garder un contact avec les partenaires des hommes violents durant le traitement afin d'assurer leur sécurité, de les prévenir de menaces proférées à leur égard durant les sessions et de les aviser lorsque l'homme violent quitte le groupe. Certains organismes engagent une personne ressource pour effectuer ce travail.

L'équipe devrait être composée de deux personnes, une femme et un homme. Cette formule permet aux hommes violents de voir et de comprendre qu'il est possible pour un homme et une femme de bien travailler ensemble, de partager des responsabilités également afin d'atteindre un but commun.

Je crois que des frais minimes devraient être imposés aux hommes à chaque session afin de les responsabiliser face à leur comportements. Certains organismes offrent aux hommes qui sont incapables de payer d'effectuer du travail communautaire.

Certains hommes violents devraient être exclus des programmes. Les problèmes liés à la consommation d'alcool et de drogues devraient être réglés avant que l'homme puisse participer aux sessions. Il va de soi que l'agressivité envers les intervenantes et les intervenants ne doit pas être tolérée. Les hommes ayant des problèmes de santé mentale seraient aussi exclus. Et il est d'importance primordiale que les hommes aient accepté la responsabilité de leur comportement violent avant de commencer les sessions.

Ce dernier point explique pourquoi il est difficile d'aider les hommes qui sont référés par un décret légal. La décision de participer au programme n'ayant pas été prise de leur plein gré, leur intérêt dépend souvent des répercussions sociales de leur arrestation (pertes dans la société). Puisque ces hommes sont mandatés par la cour criminelle, les centres qui choisissent de les accueillir doivent collaborer avec les systèmes juridique et policier et les intervenantes et intervenants doivent communiquer régulièrement avec les contrôleurs judiciaires. 
Par contre, les observations des intervenantes de la maison d'hébergement de notre région ainsi que d'autres organismes suggèrent que la participation au traitement pour un homme violent soit plus efficace lorsque sa partenaire fait aussi partie d'un groupe pour femmes victimes de violence. Ceci me porte à suggérer que dans de telles circonstances, l'homme violent qui manifeste un désir de participer à un programme devrait être accepté de façon prioritaire.

\section{Comme intervenant / intervenante}

La collaboration entre les organismes d'une même communauté devrait être encouragée et concrétisée par l'entremise d'un comité communautaire. La mise sur pied d'un comité composé de membres des centres de santé, de la cour judiciaire, du corps policier, et des maisons d'hébergement pour femmes offre un mécanisme de coordination, de concertation et d'éducation communautaire efficace pour agir contre la violence.

L'organisation et la préparation, tant au niveau des sessions que des contacts avec les conjointes afin s'assurer leur sécurité, constituent un autre aspect important du travail des intervenants et intervenantes auprès des hommes violents. Lors des sessions de groupe, la confrontation constitue un outil essentiel, puisque les hommes doivent être confrontés à leurs comportements abusifs inacceptables et à la responsabilité de changement qui leur incombe. Les personnes qui interviennent auprès des hommes violents devraient toujours se comporter d'une manière assurée afin d'éviter la manipulation de la part des hommes violents. Il est vrai que ces hommes ont besoin de soutien et d'encouragement pour changer mais leur violence ne devrait jamais être excusée, ni justifiée même s'ils ont vécu de grandes difficultés dans leur jeunesse et qu'ils ont eux-mêmes été victimes et/ou témoins de violence. Leurs mauvaises expériences ne les ont pas forcés à devenir violent. Il faut que ces hommes apprennent à surmonter 
Reflets

leur colère et à communiquer. De plus, il est important de ne jamais se centrer sur le comportement de la partenaire. Il faut plutôt se concentrer sur l'homme et sur sa violence. Tous ces conseils ne sont pas faciles et le succès est lent et demande de la patience de la part de toutes les personnes engagées dans le processus.

\section{Conclusion}

Le succès du groupe dépend, en effet, de la coopération de chaque personne engagée. L'attente des groupes féministes et du gouvernement face au succès immédiat des programmes pour hommes violents est très élevée. Par contre, étant donné la nouveauté du traitement de cette problématique, il faut donner du temps pour permettre aux interventions de se développer et de s'améliorer.

La collaboration des organismes communautaires ainsi qu'une sensibilisation auprès de la communauté à propos de la violence sont d'excellents moyens qui contribuent à la diminution de la violence et au succès des programmes pour hommes violents.

Pour terminer, je propose une simple question: comment atteindre le succès de ces programmes? Je crois qu'il s'agit d'une question de courage et de volonté. S'il n'y avait pas eu de personnes qui désiraient aider les femmes victimes de violence et éliminer les stéréotypes, il n'y aurait pas eu la création de maisons d'hébergement, de programmes de prévention de la violence, etc. Mais, comment arrêter la violence chez les hommes si personne n'essaie de traiter la cause? 